\title{
THE DANGER OF THE ANOMALOUS RECURRENT LARYNGEAL NERVE
}

By John Humphreys, M.B., Ch.B.

The David Lewis Northern Hospital, Liverpool

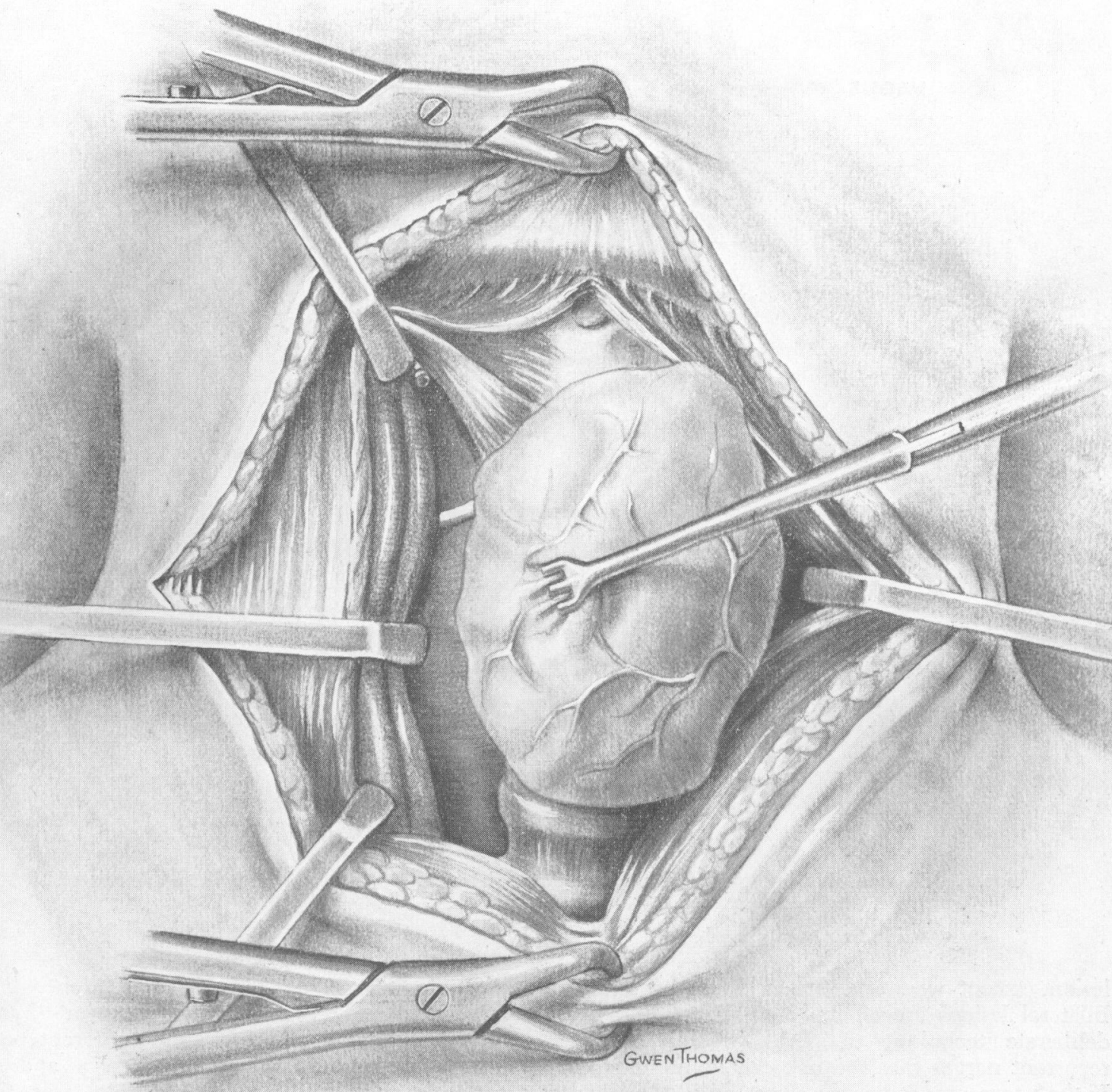

Fig. I. - Drawing of the appearance at operation. The right lobe of the thyroid is freed and rotated forwards after division of the superior pole. The anomalous nerve is seen passing from beneath the carotid sheath towards the trachea. 
Any operative procedure on the thyroid gland may endanger the laryngeal nerves. The recurrent or inferior laryngeal nerves are the most vulnerable and the consequences are more serious and permanent than in the case of the superior nerves. Routine post-operative laryngoscopy would show that unilateral recurrent nerve lesions are more common than generally supposed for compensation by the unaffected vocal cord may mask a

\section{Case Report}

Mrs. T., aged 54, was admitted to the David Lewis Northern Hospital, Liverpool, on February 22, 1954, suffering from a non-toxic adenomatous goitre first noticed four months before. Some degree of tracheal compression was noted by $\mathrm{X}$-ray.

At operation on the following day both lobes of the thyroid gland were found to be adenomatous,

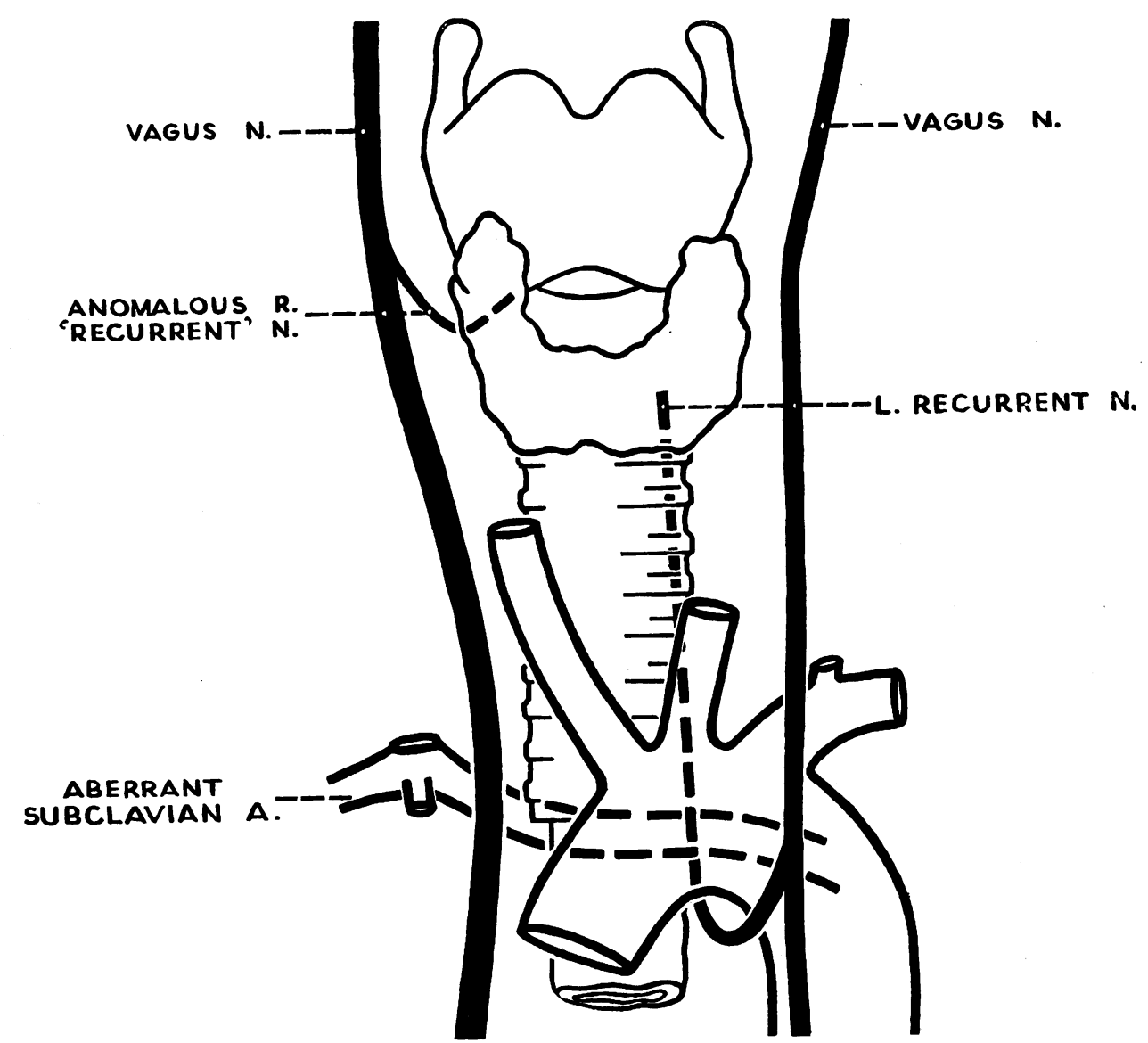

Fig. 2.-Diagram showing the probable course of the right aberrant subclavian artery and associated 'recurrent' nerve anomaly.

lesion, except when the larynx is under stress. Bilateral lesions are all too obvious. It is by a deliberate technique of demonstration of the recurrent nerves that the risk can be minimised. If the nerve cannot be demonstrated in the usual site on the right side, then the possibility of an anomalous recurrent nerve should be considered. The following case is described as an example of this anomaly. the main enlargement being in the left lobe. This lobe was mobilized first, the inferior thyroid artery ligated in continuity, the recurrent nerve demonstrated posterior to the artery and the superior pole tied. The right lobe was then mobilized, a thyroid ima artery was seen, but there was no inferior thyroid and the recurrent laryngeal nerve could not be found in the usual position. In view of the absence of the inferior 
thyroid artery, dissection was then carried out at a higher level and the nerve was seen to pass directly medially from the vagus deep to the carotid artery (Fig. I). Having reached the tracheo-oesophageal groove, it curved upwards to reach the larynx in the usual way. There was no extra laryngeal division of the nerve and it did not indent the deep surface of the gland. This nerve might easily have been ligated in mistake for a vein passing out from the gland, or for the inferior thyroid artery. The operation was then concluded by excision of the greater part of each lobe and removal of the isthmus. Post-operative demonstration of the vocal cords showed them to be moving normally. Convalescence was uneventful and the patient was discharged on the ninth post-operative day.

Developmentally the recurrent laryngeal nerve is related to the primitive fourth dorsal aortic arches. The fourth arch of the right side forms the proximal part of the right subclavian artery whilst the corresponding vessel of the left side constitutes the arch of the aorta between the origin of the left common carotid artery and the termination of the ductus arteriosus. The recurrent nerves originally pass to the larynx caudal to the sixth pair of aortic arches and are therefore dragged caudally when these descend. Thus in complete development the left nerve hooks around the ligamentum arteriosum, but on the right side the fifth and dorsal part of the sixth arches disappear and the nerve hooks around the fourth aortic arch, i.e. the commencement of the subclavian artery. If the nerve passes direct to the larynx, i.e. not pursuing a recurrent course, there must be a vascular anomaly typically of the form shown in the diagram (Fig. 2). The right subclavian artery then arises from the aorta beyond the left subclavian artery and passes behind the oesophagus. In this case this could not be demonstrated at operation but was confirmed later by a barium swallow $\mathrm{X}$-ray. This association was first demonstrated in the cadaver as early as 1823 by
Stedman and has been recognized on numerous occasions since, though rarely at operation. The nerve may easily be mistaken for the inferior thyroid artery or a middle thyroid vein, and ligated as such and possibly divided. This ligature was passed in a case described by Pemberton and Beaver (1932), in which no inferior thyroid artery was present as in the case described here. No doubt injury of the nerve in the anomalous position accounts for some cases of apparently unexplained recurrent nerve lesions, following thyroid operations.

It is not possible to assess accurately the frequency of this anomaly, but Williams (1933) has examined I59 aortic arches and four showed anomalous subclavian artery variations with a direct inferior laryngeal nerve. He suggests the surgeon may find one to four cases in every hundred thyroid operations. Only by deliberate exposure of the nerve can one be certain of avoiding injury to the recurrent nerves. The risk of damage to the nerve during dissection carried out with reasonable care is negligible; it has withstood dissection 10,000 times in the Lahey clinic without damage. Cattell (1948) has had the same experience, and by this deliberate technique has only had 33 lesions in 4,795 thyroid operations of all types; an exceptionally low incidence of 0.7 per cent. These figures serve to emphasize the value of the extra time spent on demonstration of the recurrent nerves at operation.

\section{Acknowledgments}

I express my thanks to Mr. P. R. Hawe for permission to publish this case and for his help in preparation of the paper, also Miss G. Thomas for the illustrations.

\section{BIBLIOGRAPHY}

CATTELL, R. B. (1948), West. F. Surg., 56, 77. LAHEY, F. H. (1939), Surg. Clin. N. Amer., 19, 565. LAHEY, F. H. (1944), Surg. Gynec. Obstet., 78, 239. PEMBERTON, J. DE J., and BEAVER, M. D. (1932), Surg. Gynec Obstet., 54, 594.

STEDMAN, G. W. (1823), Edin. Med. Surg. F., 19, 24. WILliaMS, G. D. (1933), Ann. Surg., 97, 828. 